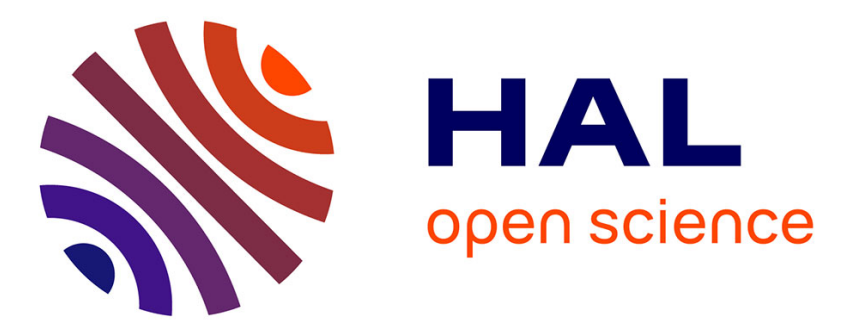

\title{
Rôle de la langue maternelle et de l'origine sociale des élèves sur l'activité de co-révision à distance d'un texte explicatif en Français Langue Seconde dans le contexte diglossique d'Haïti
}

Emilien Duvelson

\section{To cite this version:}

Emilien Duvelson. Rôle de la langue maternelle et de l'origine sociale des élèves sur l'activité de co-révision à distance d'un texte explicatif en Français Langue Seconde dans le contexte diglossique d'Haïti. Contextes et Didactiques, 2014, Éducation et socialisation en contextes multilingues et pluriculturels, 5. hal-02050135

\section{HAL Id: hal-02050135 \\ https://hal.univ-antilles.fr/hal-02050135}

Submitted on 26 Feb 2019

HAL is a multi-disciplinary open access archive for the deposit and dissemination of scientific research documents, whether they are published or not. The documents may come from teaching and research institutions in France or abroad, or from public or private research centers.
L'archive ouverte pluridisciplinaire HAL, est destinée au dépôt et à la diffusion de documents scientifiques de niveau recherche, publiés ou non, émanant des établissements d'enseignement et de recherche français ou étrangers, des laboratoires publics ou privés.

\section{(ㄷ)(1) $\$$}

Distributed under a Creative Commons Attribution - NonCommerciall 4.0 International 


\title{
Rôle de la langue maternelle et de l'origine sociale des élèves sur l'activité de co-révision à distance d'un texte explicatif en Français Langue Seconde dans le contexte diglossique d'Haïti
}

\author{
Emilien DUVELSON \\ Equipe TICE, Contexte, Langage et Cognition, Laboratoire CHArt, \\ Université de Paris 8
}

\section{Résumé}

La recherche que nous présentons vise à étudier l'effet de la langue (Créole vs Français), utilisée dans les textes d'aide, sur la compréhension et la réécriture de textes en langue seconde (français) dans le contexte diglossique d'Haïti chez des lecteurs d'origines sociales différentes. Dans la lignée des travaux sur la co-écriture de textes à distance (Legros, Makhlouf et Maître de Pembroke, 2003 ; Legros, Noyau, Cordier et Khalis, 2003 ; Gabsi, 2004 ; Hoareau, Legros, Gabsi, Makhlouf, et Khebbeb, 2006), nous avons mené notre recherche avec des enfants de CM2 âgés de 9 à 16 ans afin d'examiner les hypothèses suivantes : (i) l'utilisation du texte d'aide en langue maternelle permet la réactivation des connaissances et la replanification au cours de la révision et de la réécriture de texte en langue seconde sous forme d'informations retraitées (Scardamalia et Beraiter, 1986); (ii) l'utilisation du texte d'aide en langue seconde favorise la production d'informations issues directement du texte d'aide sans retraitement (Scardamalia et Beraiter, 1986). Les résultats apportent des informations utiles à la compréhension de l'effet de la langue maternelle utilisée dans l'apprentissage/enseignement en L2 dans le contexte diglossique d'Haïti et ils montrent que la langue L2 qui est la langue d'enseignement est aussi la langue d'apprentissage. Dans le contexte diglossique d'Haïti, la langue maternelle ne semble pas exercer le même effet sur l'apprentissage en L2, contrairement aux autres contextes plurilingues.

\section{Mots-clés}

Compréhension, production de texte, contexte diglossique.

\begin{abstract}
The current research aims to study the effect of the language (French vs Creole) used in the text guides on understanding and rewriting second language texts (French) in the diglossia context of Haiti among different social backgrounds readers. In line with the work on co-writing distance texts (Legros, Makhlouf, Maître de Pembroke, 2003 ; Legros, Noyau, Cordier et Khalis, 2003 ; Gabsi 2004 ; Hoareau, Legros, Gabsi, Makhlouf et Khebbeb, 2006), we conducted our research with children of 5th grade (CM2), aged from 9 to 16 years old, to examine the following assumptions : (i) the use of the help text in Native language allows reactivation of knowledge and re-planning during the review and the rewriting text in second language as restated informations (Scardamalia et Beraiter, 1986); (ii) the use of the help text in second language promotes the production of information coming directly without restatement of the help text (Scardamalia et Beraiter, 1986). The results provide useful information for understanding the effect of the Native language used in learning/teaching L2 in the context diglossia in Haiti and they show that the L2 that is the language of instruction is also the learning language. In the context of Haiti diglossia, the Native language does not seem to have the same effect on L2 learning, unlike other multilingual conte.
\end{abstract}

\section{Keywords}

Comprehension, text production, diglossia context. 


\section{Objectif et cadre théorique}

Cette recherche vise à étudier l'effet de l'origine socioculturelle des élèves sur la production de texte et la co-révision à distance ${ }^{1} \mathrm{~d}^{\prime}$ un texte explicatif en langue seconde (L2). L'objectif est d'analyser chez des élèves d'Haïti d'origines sociales différentes l'effet de la langue (créole versus français) utilisée dans des textes d'aide sur la co-révision à distance.

Des recherches ont montré que les activités de co-révision conduisaient à des performances supérieures à celles constatées en situation de révision monogérée (Cameron, Edmunds, Wigmore et Hunt, 1997 ; Marin et Legros, 2006). Lorsque les élèves révisent leur propre texte, les résultats s'avèrent moins satisfaisants que lorsqu'ils révisent le texte d'un pair. Gabsi en 2004 (voir Legros, Makhlouf, Gabsi et Hoareau, 2006) a mis en évidence l'effet de la langue maternelle sur le développement des compétences en co-révision en L2 et sur l'amélioration de la qualité des textes produits (van Weijen, van den Bergh, Rijlaarsdam et Sanders, 2009). Plus précisément, Gabsi (2004) visait à étudier le rôle de la langue maternelle (tamazight) utilisée dans un texte d'aide sur la révision et la co-révision à distance d'un texte explicatif en L2 (français) et sur la (co)construction des connaissances, en situation plurilingue et pluriculturelle. Dans ce but, elle a utilisé le paradigme de la révision et de la réécriture de texte afin d'étudier les effets de l'interaction de la langue maternelle (L1) et de la langue seconde (L2) sur la production de texte en contexte plurilingue. Deux groupes d'élèves d'un collège de Meudon et deux groupes d'un collège de la wilaya de Tizi Ouzou ont participé à l'expérimentation. Dans une première séance, les élèves ont produit, en français, un premier jet d'un texte explicatif sur les causes et les conséquences de la pollution des eaux douces, suivi d'une tâche de lecture d'un texte informatif. Le but de la lecture de ce texte était d'aider les élèves, lors de la révision, à replanifier le $1^{\text {er }}$ jet de leur texte et à enrichir son contenu sémantique et sa forme. Le texte a été présenté au premier groupe (G1) d'élèves de la wilaya de Tizi Ouzou en langue maternelle, le tamazight, et au second groupe en français (G2). Les mêmes tâches ont été proposées aux deux groupes de collégiens français avec la lecture du texte d'aide en français. L'ensemble des participants ont réécrit leur texte d'abord individuellement (Réécriture 1) et, quelques jours plus tard, en binômes constitués d'un collégien algérien et d'un collégien français (Réécriture 2). Deux questionnaires de connaissances (initial et final) ont encadré les tâches de production et de réécriture. Le but était d'analyser, en comparant les réponses aux questionnaires initial et final, l'effet de la langue lors de l'activité de production et de co-révision sur la modification des représentations des connaissances initiales.

La première hypothèse générale est que l'analyse des protocoles et, en particulier, la comparaison entre les groupes G1 et G2 des informations ajoutées au $1^{\mathrm{er}}$ écrit lors de la révision (Réécriture 1) et au deuxième écrit, lors de la co-révision (Réécriture 2), révèlent une différence quantitative et qualitative des ajouts que l'auteur attribue à un effet de la langue maternelle utilisée dans les textes d'aide (G1) sur les processus de révision et de co-révision et sur la réorganisation des connaissances en mémoire. La langue maternelle, en facilitant l'activation des connaissances en mémoire, influencerait la qualité du fonctionnement de la mémoire, lors de la relecture, du retraitement du $1^{\mathrm{er}}$ jet, et des réécritures 1 et 2 (Hoareau et Legros, 2005, 2006).

\footnotetext{
${ }^{1}$ Il s'agit dans ce cas d'une distance socioculturelle.
} 
La seconde hypothèse générale découle de la première et consiste à supposer un effet de la langue sur l'activation des connaissances en mémoire sur les réponses au second questionnaire et donc une amélioration sur l'activation et la réorganisation des connaissances en mémoire.

Les résultats indiquent que l'utilisation de la langue maternelle (L1) dans les textes d'aide favorise l'activation des connaissances et par conséquent facilite la tâche de réécriture 1 en français (L2). Les participants qui ont lu le texte d'aide en langue maternelle (L1) produisent un texte plus complet par le nombre de propositions ajoutées et plus précis par le nombre d'informations issues du modèle de situation sous-jacent au texte, que ceux qui utilisent uniquement la langue L2 (voir Hoareau et Legros, 2005). Ce sont les informations issues des connaissances évoquées par le texte (modèle de situation) et activées grâce à l'utilisation de la langue maternelle qui permettent en effet de produire un texte de meilleure qualité. Lorsque les élèves ont lu le texte en langue L1 (G1), et qu'ils réécrivent leur propre texte (Réécriture 1), ils enrichissent davantage le contenu de leur texte et ajoutent beaucoup plus d'informations à leur premier jet que les élèves qui ont lu le texte en L2 (G2). En revanche, lorsque les élèves révisent le texte de leur partenaire, ils ajoutent plus d'informations renvoyant au modèle de situation, mais aussi ils traitent mieux la structure de surface du texte.

Hoareau, Legros, Gabsi, Makhlouf et Khebbeb (2006) ont réanalysé les données de Gabsi (2004) dans le but d'interpréter ces effets en fonction du modèle de l'expertise d'Erickson et Kintsch (1995). Selon ces derniers, le niveau d'expertise des individus dans un domaine de connaissance donné exerce un effet sur leur capacité de compréhension et de rappel d'un texte traitant de ce domaine. Ericsson et Kintsch (1995) conçoivent une composante de la mémoire à long terme (MLT) appelée Mémoire de Travail à Long Terme (MTLT) qui constitue la partie active de la MLT. Ils s'appuient sur des données provenant de travaux conduits sur l'étude des capacités de compréhension et de rappel d'individus experts dans un domaine de connaissance donné, et ils constatent que leurs résultats sont supérieurs à ceux d'individus non experts. Contrairement aux prédictions issues des modèles classiques de la mémoire, le modèle d'Ericsson et Kintsch (1995) peut rendre compte des phénomènes observés. Hoareau et al. (2006) ont validé l'hypothèse du rôle de la Mémoire de Travail à Long Terme sur l'activation des connaissances. Lorsque les sujets possèdent des connaissances sur le domaine évoqué par le texte, et notamment lorsque ces connaissances construites dans la langue maternelle sont activées - les sujets qui utilisent la langue maternelle sont experts en langue maternelle - les structures de rappel élaborées et/ou activées permettent un fonctionnement optimal de la Mémoire de Travail à Long Terme (MTLT) et donc une meilleure activation des connaissances et une meilleure production de textes. Hoareau et al. (2006) concluent que la langue maternelle en contexte diglossique exerce un effet sur la qualité du fonctionnement des structures de rappel de la Mémoire de Travail à Long Terme (MTLT) et donc sur la qualité du traitement inférentiel lors de la replanification et de la réécriture d'un texte explicatif en L2. Plus précisément, ils montrent qu'après la lecture d'un texte d'aide en langue maternelle (L1) les participants activent davantage leurs structures mémorielles et donc améliorent le retraitement au cours de la replanification et la réécriture.

La mémoire de Travail à Long-Terme (MTLT) qui constitue la partie active de la MLT fonctionne dans les domaines où le sujet possède un niveau de connaissance élevé. Elle est constituée de structures de rappel (informations inter-connectées en MLT), (voir Figure 1). 


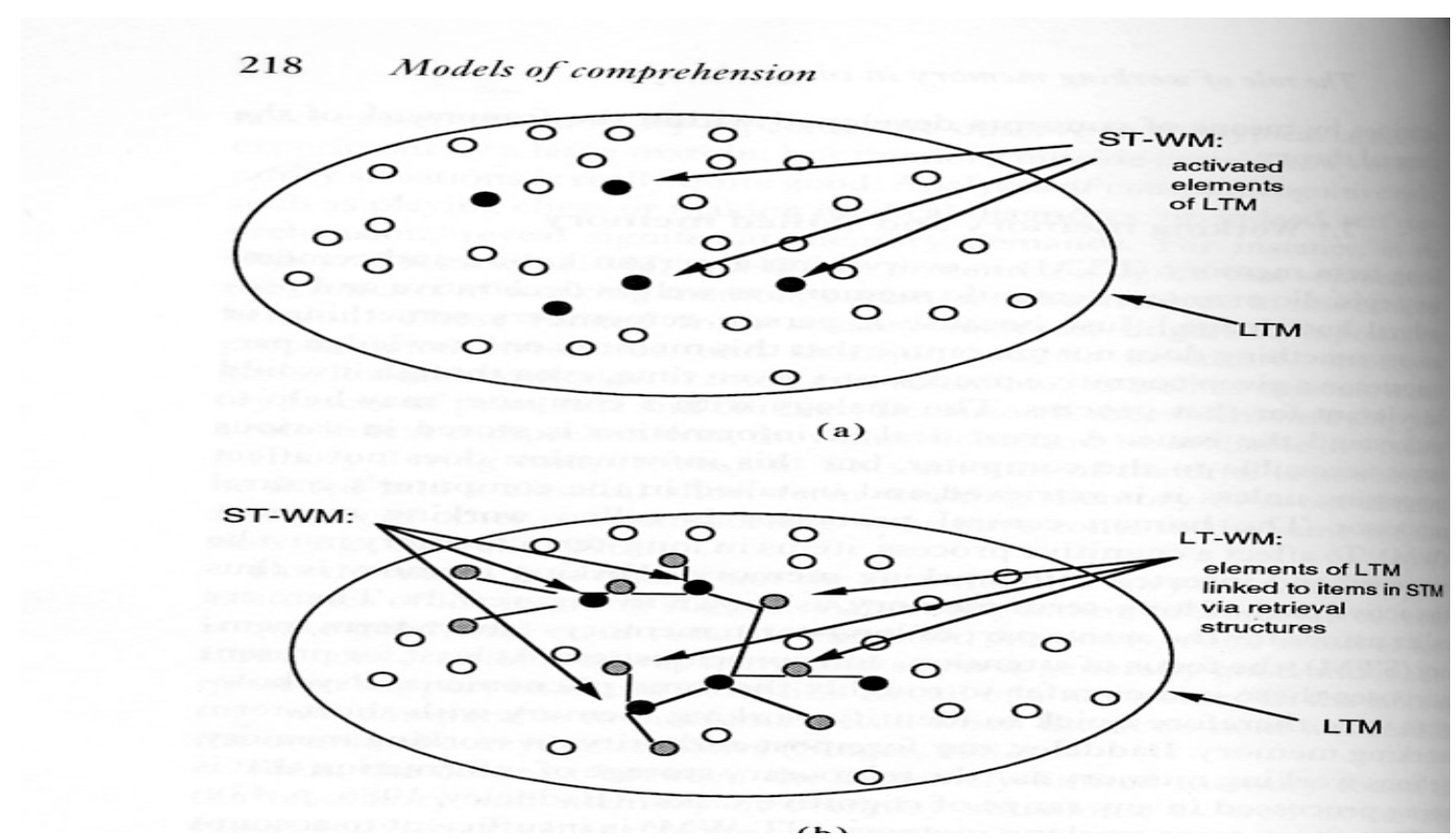

Figure 1 : La Mémoire de Travail à Long Terme, in W. Kinsch (1998)

Dans l'expérience que nous avons conduite en Haïti, nous avons demandé à deux groupes de participants de niveau socioculturel différent : G1 (enfants de milieux défavorisés, fréquentant les écoles publiques et parlant le créole à la maison) et G2 (enfants de milieux favorisés, fréquentant les écoles privées et parlant essentiellement le français à la maison) de produire une première version d'un texte explicatif en français (L2). La lecture d'un texte documentaire a été ensuite proposée afin d'aider les élèves à réviser leur écrit. Ce texte était présenté dans deux sous groupes d'enfants défavorisés (G1) et favorisés (G2) en langue maternelle L1 (créole) et en langue seconde L2 (français). Les enfants devaient lire le texte et prendre des notes. Peu après, les élèves ont effectué une tâche de révision de leur $1^{\text {er }}$ écrit (Réécriture 1). Quelques jours plus tard, les enfants de milieux défavorisés (G1) ont révisé le premier jet des enfants de milieux favorisés (G2) qui eux aussi ont révisé le premier jet de ceux de milieux défavorisés (Réécriture 2).

Deux questionnaires de connaissances (initial et final) ont encadré les deux tâches de production (écriture et réécriture). En nous appuyant sur la théorie de la Mémoire de Travail à Long Terme, nous supposons que les élèves « experts » dans une langue activeront plus de connaissances que les non experts dans cette langue. Dans la mesure où les élèves de milieux défavorisés sont experts dans la langue L1, nous supposons qu'ils seront capables d'activer les structures de connaissances construites dans cette langue leur permettant un fonctionnement optimal de la mémoire à long terme et donc qu'ils seront capables de produire un texte d'aussi bonne qualité que les élèves de milieux favorisés plus experts en L2.

Dans le cadre de cet article, nous présentons tout d'abord les résultats de l'analyse de la réécriture 2 et nous testons l'effet de la langue (L1 vs L2) utilisée dans le texte d'aide sur le type d'information ajoutée c'est-à-dire sur la façon dont les informations ajoutées sont traitées. Elles peuvent en effet être simplement extraites du texte d'aide et « collées » au texte produit 
(knowledge telling strategy) ou extraites des connaissances activées lors de la lecture du texte d'aide et issues de la mémoire de travail à long terme (connaissances «transformées », ou knowledge transforming strategy) et résultant d'un retraitement sémantique (Crinon et Legros, 2002 ; Scardamalia et Bereiter, 1986). Nous présentons ensuite les résultats de l'analyse des réponses au second questionnaire afin de rendre compte de l'effet du niveau socioculturel des élèves et donc de la langue utilisée dans les textes d'aide sur la réorganisation des connaissances en mémoire.

\section{Principales hypothèses}

\subsection{Hypothèse 1. Effet de la langue ( $L 1$ vs L2) sur le type de traitement [Copié (T1) vs Transformé (T2)]}

Nous avançons l'hypothèse que le texte d'aide en langue L1 proposé aux enfants défavorisés (G1) facilite davantage la production d'ajouts "transformés » (T2) que d'ajouts « copiés » (T1), alors que la lecture en langue L2 favorise la production d'ajouts « copiés » (T1).

\subsection{Hypothèse 2. Effet de la langue (L1 vs L2) sur le type de proposition ajoutée au questionnaire final.}

Nous proposons l'hypothèse que lors du questionnaire final, les élèves de milieux défavorisés (G1) qui lisent le texte d'aide en langue maternelle, ajoutent aux réponses au questionnaire initial plus d'informations issues des connaissances stockées en mémoire à long terme (Q5 et

$\mathrm{Q6}^{2}$ ). En revanche, lorsqu'ils lisent le texte en langue L2, ils ajoutent plus d'informations issues du texte d'aide et renvoyant à la microstructure de ce texte (informations peu importantes (Q1 et Q2) ou à sa macrostructure (Q3 et Q4)).

\section{Méthode}

\subsection{Participants}

Les participants sont des élèves de CM2 d'Haïti issus de deux milieux sociaux distincts : défavorisé (G1) et favorisé (G2). Chaque groupe est divisé en deux sous-groupes selon la langue utilisée dans le texte d'aide :

- Groupe 1: milieux défavorisés

G1.1 : 25 Sujets (texte d'aide à la réécriture en créole)

G1.2 : 25 Sujets (texte d'aide à la réécriture en français)

- Groupe 2 : milieux favorisés

G2.1 : 25 Sujets (texte d'aide à la réécriture en créole)

$\mathrm{G} 2.2: 25$ sujets (texte d'aide à la réécriture en français)

- Groupe 3 : groupe témoin (les deux milieux)

G3.1 : 15 sujets de milieux défavorisés (groupe témoin : pas de texte d'aide)

G3.2 : 15 sujets de milieux favorisés (groupe témoin : pas de texte d'aide)

\footnotetext{
${ }^{2}$ Voir page suivante : matériel expérimental.
} 


\subsection{Matériel expérimental}

Le matériel expérimental est composé d'un questionnaire et d'un texte explicatif d'aide à la réécriture.

\subsubsection{Questionnaire}

Le questionnaire proposé en pré- et en post-test porte sur le domaine de connaissances : les causes et les conséquences du changement climatique. Il est composé de 6 questions. Ces questions permettent d'activer trois types de représentations telles qu'elles sont conceptualisées par van Dijk et Kintsch (1983).

Les réponses aux questions Q1 et Q2 sont constituées d'informations renvoyant à la microstructure du texte, sans liens de causalité. Ce sont les informations les plus faciles à produire et qui relèvent d'un simple rappel.

Les réponses aux questions Q3 et Q4 sont constituées d'informations reliées par des liens de causalité renvoyant à la macrostructure et faisant l'objet d'un traitement inférentiel.

Les réponses aux questions Q5 et Q6 sont des informations appartenant au modèle de situation du texte produit et qui résultent d'un traitement inférentiel élargi aux connaissances générales reliées au topic précis du texte produit.

1. Quels sont les types d'activités humaines qui peuvent contribuer au changement du climat?

2. Quelles sont les autres causes du changement climatique que les activités humaines?

3. Quelles conséquences le changement climatique peut-il avoir sur la nature?

4. Pourquoi le climat change-t-il ?

5. Quels sont les gestes au quotidien qui permettent de lutter contre le changement climatique?

6. Comment la température du climat peut-elle augmenter?

\subsubsection{Texte explicatif (extrait des deux versions $d u$ texte)}

Le texte proposé, dans le cadre de cette expérimentation, porte sur les causes et les conséquences du changement climatique. Deux versions de ce texte ont été utilisées, une version en français L2 et une version en créole L1 (langue maternelle des participants). 


\section{Version L2}

Le changement du climat est dû à deux facteurs naturels : les variations de la quantité d'énergie solaire reçue à la surface de la Terre et les variations de la trajectoire de la Terre autour du Soleil. Mais en plus de cette évolution naturelle, le climat est de plus en plus influencé par les activités polluantes des hommes. Le climat a toujours évolué depuis la formation de la Terre, il y a 4,5 milliards d'années. Les fluctuations du climat passé sont donc normales et naturelles. Il y a eu des périodes glaciaires très froides et très longues suivies par des périodes interglaciaires plus chaudes mais plus courtes. La dernière période interglaciaire a eu lieu il y a 120000 ans. La température était alors plus basse qu'au début des années 2000. Le niveau de la mer était de 120 mètres inférieur à celui d'aujourd'hui...

\section{Version $L 1$}

Chanjman klima a gen de (2) faktè natirèl ki lakoz : premyèman se kantite ènèji solèy la ki rive sou tè a; answit se modifikasyon tè a genyen lè 1 ap vire toutotou solèy la. Anplis faktè natirèl sa yo, klima kounye a sibi anpil enfliyans moun kap viv sou tè a. Klima a te toujou konn chanje depi byen lontan, sa gen 4,5 milya lane. Men chanjman sa yo te nòmal epi natirel. Te konn gen tan fredi kote li fè frèt anpil epi ki te long tou, aprè sa te konn gen peryòd ki cho epi ki te kout. Dènyè peryòd entèglasè kote li te frèt anpil, sa gen 120000 lane. Lè sa, tanperati a te pi ba pase kòmanseman lane 2000. Lanmè a te 120 mèt pi ba pase jounen jodi a...

\subsection{Procédure expérimentale}

Lors d'une première séance, les participants répondent à un questionnaire de connaissance initial (10 minutes). Au cours d'une deuxième séance, ils produisent le premier jet d'un texte explicatif en français L2 (30 minutes). Quelques jours plus tard, les participants des deux groupes lisent un texte d'aide, soit en créole soit en français, et prennent des notes. Ils réécrivent ensuite leur propre texte à partir de leur premier jet (Réécriture 1). La première réécriture est suivie d'une seconde réécriture où les participants de chaque groupe ont révisé chacun le texte d'un partenaire distant (Réécriture 2). Au cours d'une dernière séance, l'ensemble des participants répond au questionnaire final.

\subsubsection{Variables dépendantes}

La variable dépendante prise en compte dans cette étude est le type de propositions ajoutées (T1 = copiées $v s \mathrm{~T} 2=$ transformées) par les deux populations d'élèves. Concernant le questionnaire final, nous avons pris en compte le nombre de propositions ajoutées en termes d'ajouts renvoyant à la microstructure (informations peu importantes, MICRO), à la macrostructure (informations importantes, MACRO) et au modèle de situation (informations issues des connaissances évoquées par le texte MS). 


\section{Principaux résultats}

4.1. Analyse 1 : Effet de la langue utilisée dans les textes d'aide (L1 vs L2) sur la replanification et la réécriture 2 d'un texte explicatif en contexte diglossique, en fonction de l'origine socioculturelle des élèves

Pour chaque groupe, nous analysons tout d'abord les informations ajoutées en fonction du type de traitement mis en œuvre : (i) informations ajoutées « retraitées » (knowledge transforming strategy), absentes du texte d'aide, mais activées lors de la lecture de ce texte ; (ii) informations « copiées » du texte source et collées sans modifications lors de la réécriture (knowledge telling strategy).

Les données ont été analysées selon le plan $\underline{\mathrm{S}}<\mathrm{G} 3 * \mathrm{~L} 2>^{*} \mathrm{~T} 2$ dans lequel les lettres $\mathrm{S}, \mathrm{G}, \mathrm{L}, \mathrm{T}$ renvoient respectivement aux facteurs Sujet (facteur aléatoire), Groupe (G1 = groupe défavorisé ; G2 = groupe favorisé ; G3 = groupe témoin), Langue (L1 = Langue maternelle ; L2 $=$ langue seconde), Types de Traitement des propositions ajoutées $(\mathrm{T} 1=$ Ajouts Copiés, $\mathrm{T} 2=$ Ajouts Transformés).

Les enfants de milieux défavorisés (G1) produisent plus d'ajouts «traités » renvoyant à la stratégie des connaissances transformées $(11,76)$ que d'ajouts « collés » renvoyant à la stratégie des connaissances racontées $(8,800)$ lorsqu'ils lisent le texte d'aide en langue maternelle $(\mathrm{F}(2,124)=11,947, \mathrm{p}<.0001)$. Les élèves de milieux favorisés produisent plus d'ajouts traités $(13,080)$ que d'ajouts collés $(7,600)$ lorsqu'ils lisent le texte d'aide en langue maternelle; et ils produisent plus d'ajouts traités $(16,48)$ que d'ajouts collés $(8,200)$ lorsqu'ils lisent le texte d'aide en langue seconde L2. La langue maternelle utilisée dans les textes d'aide par les enfants défavorisés favorise le retraitement sémantique lors de la replanification de leur texte et donc l'amélioration de la qualité de leur texte. Les enfants de milieux favorisés améliorent la qualité de leur texte lors de la réécriture, quelle que soit la langue utilisée dans les textes d'aide.

\begin{tabular}{|l|c|c|c|c|c|c|c|c|}
\hline & \multicolumn{2}{|c|}{ G1L1 } & \multicolumn{2}{c|}{ G1L2 } & \multicolumn{2}{c|}{ G2L1 } & \multicolumn{2}{c|}{ G2L2 } \\
\cline { 2 - 9 } & Mean & Std & Mean & Std & Mean & Std & Mean & Std \\
\hline T1 & 8,800 & 3,014 & 10,72 & 3,260 & 7,600 & 5,172 & 8,200 & 3,342 \\
\hline T2 & 11,76 & 2,278 & 11,48 & 2,104 & 13,080 & 4,212 & 16,48 & 3,820 \\
\hline
\end{tabular}

Tableau 1 : Moyennes et écarts types des informations ajoutées par les deux groupes lors de la réécriture 2 , en fonction de la langue utilisée dans les textes d'aide 


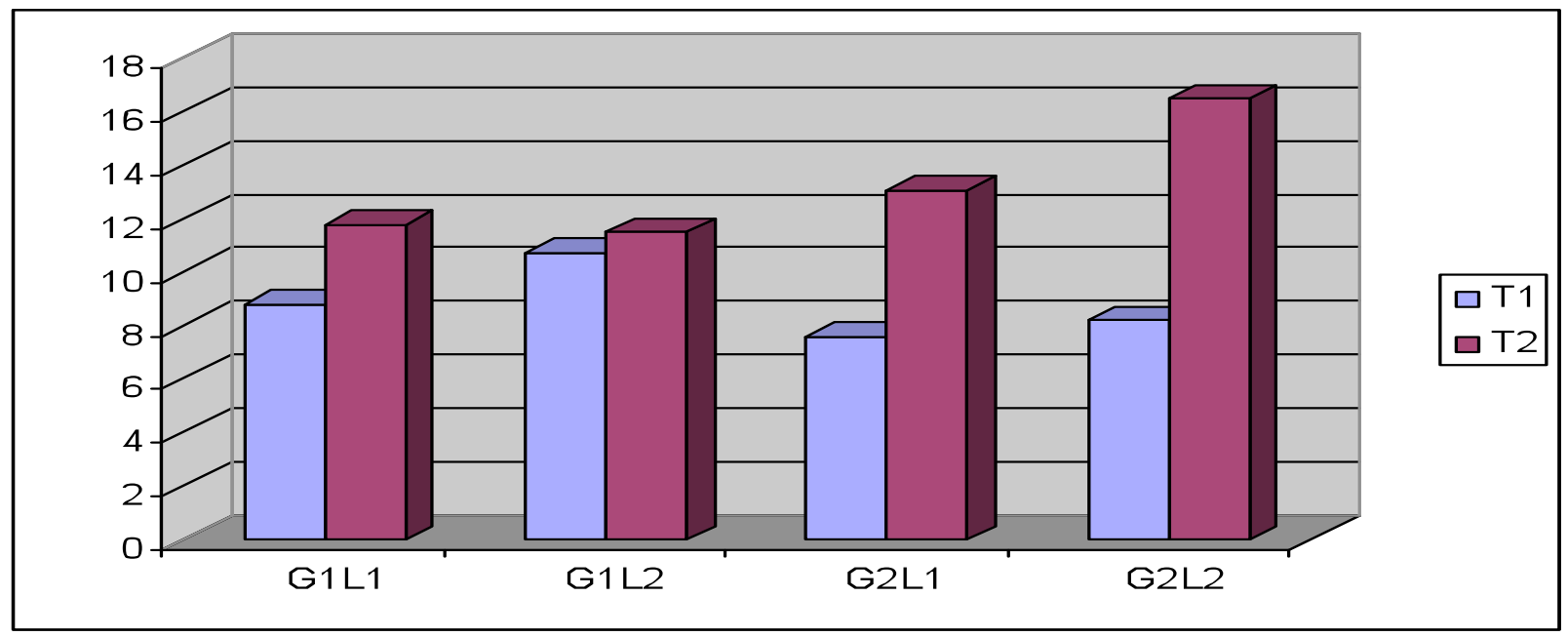

Figure 2 : Moyennes des informations ajoutées par les deux groupes en fonction de la langue utilisée dans les textes d'aide

\subsection{Analyse 2 : Effet de la langue utilisée sur les réponses au questionnaire final}

Les réponses aux questions ont été analysées selon le plan : $\underline{\mathrm{S}}<\mathrm{G} 3{ }^{*} \mathrm{Q} 6$ dans lequel les lettres $\mathrm{S}, \mathrm{G}$ et $\mathrm{Q}$ renvoient respectivement aux facteurs Sujet (facteur aléatoire); Groupe (G1 = groupe de sujets de milieux défavorisés; $\mathrm{G} 2$ = groupe de sujets de milieux favorisés ; G3 = groupe témoin); Questions Q1, Q2 (réponses renvoyant à la microstructure), Q3, Q4 (réponses renvoyant à la macrostructure) et Q5, Q6 (réponses renvoyant au modèle de situation).

Les élèves de milieux défavorisés qui lisent le texte d'aide en langue maternelle produisent plus d'ajouts aux réponses aux questions renvoyant au modèle de situation évoqué par le texte $(19,200)$ qu'à celles renvoyant à la microstructure $(13,400)$ et à la macrostructure $(15,840)$, $\mathrm{F}(4,248)=8,057, \mathrm{p}<.0001$. De même, lorsqu'ils lisent le texte d'aide en langue seconde, ils produisent plus d'ajouts aux questions renvoyant au modèle de situation $(15,360)$ qu'à celles renvoyant à la micro $(11,680)$ et à la macrostructure $(12,080)$. Les élèves de milieux défavorisés ont des performances équivalentes à celles des élèves de milieux favorisés. En effet, les élèves de milieux favorisés qui lisent le texte d'aide en langue maternelle produisent plus d'ajouts aux questions renvoyant au modèle de situation $(19,120)$ que d'ajouts renvoyant à la micro $(15,800)$ et à la macrostructure $(17,600)$. Lorsqu'ils lisent le texte d'aide en langue seconde, ils produisent plus d'ajouts liés au modèle de situation $(17,280)$ que d'ajouts renvoyant à la micro $(8,840)$ et à la macrostructure $(10,960)$. Ces résultats indiquent que la langue maternelle contribue à activer chez les élèves de milieux défavorisés leurs connaissances construites dans cette langue et ils peuvent traiter l'information de la même manière que les enfants favorisés, ce qui explique leurs performances similaires à celles des enfants de milieux défavorisés lors de la réécriture 2. 


\begin{tabular}{|c|c|c|c|c|c|c|c|c|}
\hline \multirow{2}{*}{} & \multicolumn{2}{|c|}{ G1L1 } & \multicolumn{2}{c|}{ G1L2 } & \multicolumn{2}{c|}{ G2L1 } & \multicolumn{2}{c|}{ G2L2 } \\
\cline { 2 - 9 } & Mean & Std & Mean & Std & Mean & Std & Mean & Std \\
\hline Micro & 13,400 & 3,512 & 11,680 & 2,322 & 15,800 & 2,102 & 8,840 & 3,096 \\
\hline Macro & 15,840 & 2,055 & 12,080 & 2,080 & 17,600 & 2,160 & 10,960 & 4,198 \\
\hline MS & 19,200 & 2,327 & 15,360 & 2,827 & 19,120 & 2,977 & 17,280 & 4,988 \\
\hline
\end{tabular}

Tableau 2 : Moyennes et écarts types des différents types de traitement en fonction des groupes et de la langue

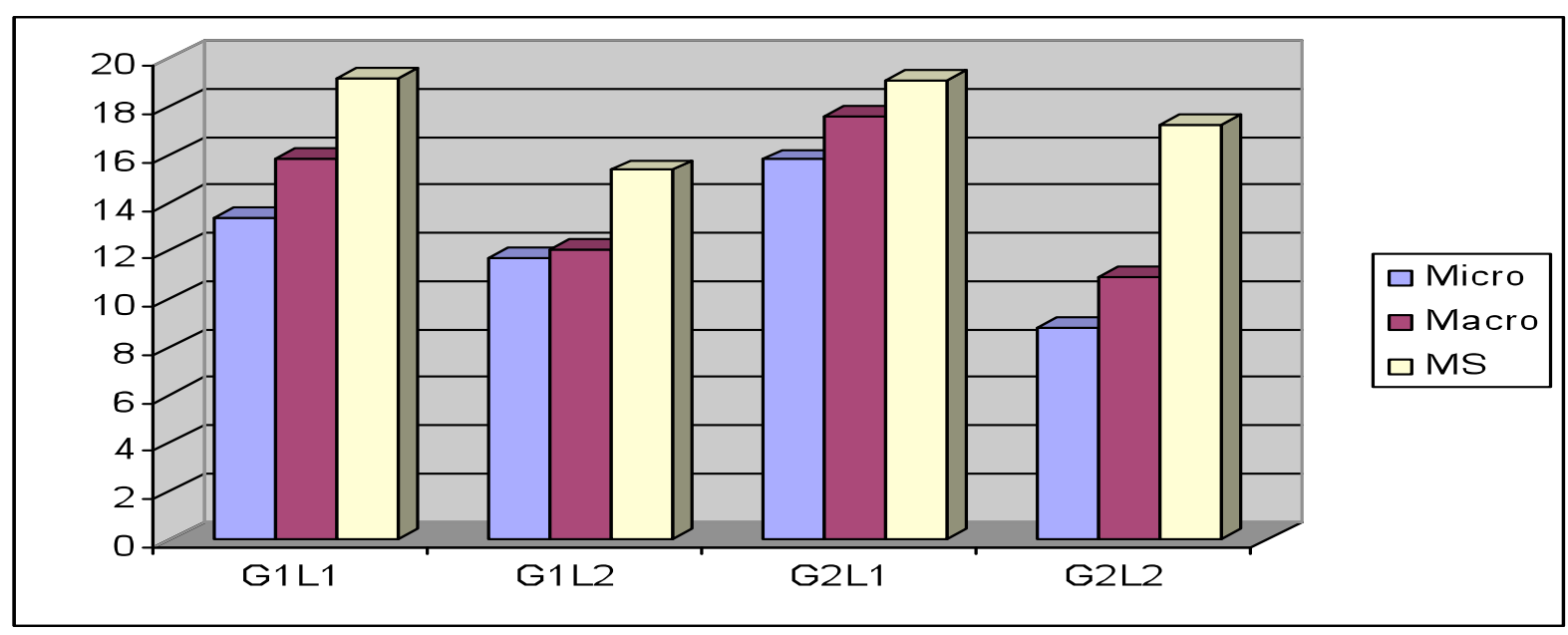

Figure 3 : Moyennes des différents types de traitement en fonction des groupes et de la langue

\section{Interprétation et perspectives}

Ces résultats mettent en évidence le rôle de la langue maternelle (créole) sur la réactivation des connaissances lors de la réécriture à distance.

L'ensemble de nos résultats nous permet d'écarter l'hypothèse d'un dysfonctionnement cognitif chez les enfants issus de milieux défavorisés lors de la conservation et de l'activation des connaissances en mémoire (Baudet, 1986). Ils montrent en effet que lorsqu'ils utilisent la langue maternelle, les élèves issus de milieux défavorisés obtiennent des performances similaires à celles des élèves de milieux favorisés.

Ces résultats apportent des informations utiles à la compréhension de l'effet de la langue maternelle utilisée dans l'apprentissage/enseignement en L2 dans le contexte diglossique d'Haïti. Dans ce contexte, la langue maternelle semble exercer un effet sur l'apprentissage en L2 qui diffère selon le milieu socioculturel des élèves. Ces résultats contribuent à enrichir les cadres théoriques de référence en mettant en évidence le rôle de la langue maternelle et de la culture dans la qualité du traitement de l'activité inférentielle et qui dépend de la qualité de fonctionnement de la Mémoire de Travail à Long Terme (MTLT) (Legros, Bounouara, Acuna, Benaïcha, Hoareau, et Sawadogo, 2009). 
L'ensemble de ces données ouvrent des perspectives sur le rôle que pourrait jouer la langue maternelle dans les situations de co-construction de connaissances à distance et dans les fondements de nouvelles approches didactiques qui prennent en compte les contextes linguistiques (Sawadogo et Legros, 2007, 2008 ; Sawadogo, Legros et Marouby, 2010).

\section{Références bibliographiques}

Baudet, S. (1986). La mémorisation de récit chez l'enfant d'âge préscolaire. Origine sociale et accès à l'information stockée en mémoire. L'année psychologique, 86, 223-246.

Cameron, C.A., Edmunds, G., Wigmore, B. et Hunt, A.K. (1997). Children's Revision of Textual Flaws. International Journal of Behavioral Development. 20(4), 667-680.

Crinon, J. et Legros, D. (2002). The Semantic Effects of Consulting a Textual Data-Base on Rewriting. Learning and Instruction, 12, 605-626.

Ericsson, K.A., et Kintsch, W. (1995). Long-term working memory. Psychological Review, $102,211-245$.

Gabsi, A. (2004). Contexte plurilingue et construction de connaissances. Co-construction de connaissances via la réécriture croisée à distance en contexte plurilingue. Mémoire de DEA non publié, Université de Paris 8, Septembre 2004. En ligne: http://classenumerique.cite-sciences.fr/numeral/textes/IMG/pdf/Amel_Manip_DEA16.pdf.

Hoareau, Y. et Legros, D. (2005). Effet de la langue maternelle (L1, Créole) sur la compréhension de texte explicatif en langue seconde (L2) en situation diglossique. Rôle de la langue L1 dans l'activation de la Mémoire de Travail à Long Terme. Communication présentée au colloque d'Appropriation du français et construction de connaissances via la scolarisation en situation diglossique, université de Nanterre, Nanterre, France, 24-26 février 2005 (TCAN/CNRS).

Hoareau, Y. et Legros, D. (2006). Rôle des contextes culturels et linguistiques sur le développement des compétences en compréhension et en production de textes en L2 en situation de diglossie. Enfance, 2, 191-199.

Hoareau, Y., Legros, D., Gabsi, A., Makhlouf, M. et Khebbeb, A. (2006). Internet et aides à la réécriture à distance de textes explicatifs en contexte plurilingue. Dans A. Piolat (dir.), Lire, Écrire, Communiquer et Apprendre avec Internet (p. 277-297). Paris : Solal. En ligne : http://classe-numerique.cite-sciences.fr/numeral/textes/IMG/pdf/

Kintsch, W. (1998). Comprehension: A paradigm for cognition. Cambridge : Cambridge University Press.

Legros, D., Maître de Pembroke, E. et Makhlouf, M. (2003). Interagir autour d'un mémoire en construction : l'exemple d'une collaboration à distance. Dans J. Crinon (dir.), Le mémoire professionnel des enseignants, observatoire des pratiques et levier pour la formation (p. 145-157). Paris : L'Harmattan.

Legros, D., Noyau, C., Cordier, M. et Khalis, A. (2003). Rôle de la langue (L1 vs L2 en situation de diglossie) et du type de questionnaire sur l'activation des connaissances dans une tâche de production en L2. Le cas des élèves du Togo. 36ème colloque international de la société européenne de linguistique, Linguistique et corpus. Types de données et comparaison des langues, Lyon, ENS-LSH.4-7 September 2003, ENS de Lyon.

Legros, D., Makhlouf, M., Gabsi, A. et Hoareau, Y. (2006). Rôle de la langue maternelle dans la production de texte en langue seconde (L2) et la construction de connaissances en 
contexte plurilingue. Statut de l'analyse du corpus et rôle des questionnaires. 30th International Conference On Functional Linguistics, University of Cyprus, 18-21 October 2006

Legros, D., Bounouara, Y., Acuna, T., Benaïcha, F.Z., Hoareau, Y. et Sawadogo, F. (2009). TICE et Cognition de la Littératie plurilingue. Vers un modèle intégrateur ${ }^{3}$. Synergies Algérie, 6, 21-28. En ligne :

http://ressources-cla.univ-fcomte.fr/gerflint/Algerie6/legros.pdf

Marin, B. et Legros, D. (2006). La prise en compte des contextes linguistiques et culturels dans les activités de révision et de co-révision à distance. Langages, 164, 113-125.

Sawadogo, F. et Legros, D. (2007). Effets des questionnements à distance via Internet sur l'activation des connaissances et l'activité de planification lors de la co-écriture de textes explicatifs en langue seconde en contexte diglossique. Conférence EIAH'2007 (Environnements Informatiques pour l'Apprentissage Humain), Lausanne (Suisse), 27-29 juin 2007.

Sawadogo, F. et Legros, D. (2008). Diversité linguistique africaine : fondements cognitifs pour une intégration des langues locales dans les nouveaux espaces collaboratifs. Universités Francophones et Diversité Linguistique, Yaounde, 27-28 juin 2008.

Sawadogo, F., Legros, D. et Marouby, G. (2010). La langue L1 dans les processus de construction de connaissance un facteur de construction identitaire et de qualité de l'éducation. Cas du Burkina et de l'Algérie. XIVème Congrès Mondial Conseil Mondial des Sociétés d'Education Comparée (WCCES), « Traçage et re-traçage des frontières et nouvelles possibilités dans l'éducation et la société ». ISTANBUL, 14-18 juin 2010.

Scardamalia, M. et Bereiter, C. (1986). Knowledge telling and knowledge transforming in written composition. Dans S. Rosenberg (dir.), Reading, writing and language learning (p. 142-175). Cambridge : Cambridge University Press.

Van Weijen, D., Van den Bergh, H., Rijlaarsdam, G. et Sanders, T. (2009). L1 use during L2 writing : An empirical study of a complex phenomenon. Journal of Second Language Writing, 18, 235-250.

\footnotetext{
${ }^{3}$ Recherche conduite dans le cadre du projet Numéral (Numérique et apprentissages locaux), Programme TCANCNRS (Technologies de la Connaissance et Apprentissages Numériques, 2004-2007).
} 